\title{
Caracterización y preparación de ceniza volante para su uso como recubrimientos cerámicos sobre substratos metálicos
}

\author{
A. CHÁVEZ, G. VARGAS, J.M. ALMANZA \\ Cinvestav-Unidad Saltillo, \\ Coah. México.
}

\begin{abstract}
Muestras de ceniza volante fueron caracterizadas por medio de absorción atómica, espectroscopía de plasma, ATD/ATG, MEB/EDXS, DRX y difracción láser con el propósito de realizar recubrimientos de ceniza volante sobre substratos de acero inoxidable por medio de la técnica de deposición electroforética. Los polvos de ceniza fueron molidos y el carbón residual y el $\mathrm{Fe}_{2} \mathrm{O}_{3}$ fueron eliminados por medio de calcinación y separación magnética, respectivamente. Se realizaron suspensiones de ceniza volante molida en los siguientes medios polares: agua, etanol, isopropanol y acetona. El pH fue controlado por medio de adición de soluciones acuosas de $\mathrm{NaOH}$ y $\mathrm{HCl}$. Los resultados de movilidad dinámica y potencial zeta efectuados a estas suspensiones por medio de la técnica electroacústica en un intervalo de $\mathrm{pH}$ de 4 a 12, mostraron que la ceniza volante presenta mejor estabilidad en acetona-solución acuosa de $\mathrm{NaOH}$. El valor más elevado de potencial zeta en este medio fue de $-50 \mathrm{mV}$. Los recubrimientos electroforéticos fueron realizados a 100, 200 y $300 \mathrm{~V}$ durante 1 a 5 minutos. Los recubrimientos caracterizados por MEB/EDX mostraron un excelente empaquetamiento de partículas y una superficie homogénea sin agrietamiento. Además el tratamiento térmico de los recubrimientos a $1050^{\circ} \mathrm{C}$ permitió una sinterización parcial de los mismos.
\end{abstract}

Palabras clave: ceniza volante, recubrimientos, suspensiones, potencial zeta.

Preparation and characterization of fly ash for use as ceramic coatings on metallic substrates.

Fly ash samples were characterized by atomic absorption, plasma spectroscopy, DTA/TGA, SEM/EDS, XRD and laser diffraction in order to obtain fly ash coatings on stainless steel substrates by electrophoretic deposition. Fly ash was milled and residual carbon and $\mathrm{Fe}_{2} \mathrm{O}_{3}$ were eliminated by calcination and magnetic separation, respectively. Fly ash suspensions were prepared with several organic media such as water, ethanol, isopropanol and acetone. The $\mathrm{pH}$ of the suspensions was controlled by the addition of $\mathrm{NaOH}$ and $\mathrm{HCl}$ aqueous solutions. The results of dynamic mobility and zeta potential in a range of $\mathrm{pH}$ from 4 to 12 showed that fly ash has better stability in acetone- $\mathrm{NaOH}$ aqueous solution. The greatest value of zeta potential in this medium was $-50 \mathrm{mV}$. The electrophoretic coatings were carried out at 100, 200 and $300 \mathrm{~V}$ during 1 to 5 minutes. The coatings characterized by SEM/EDS, showed an excellent particle packing and a homogenous surface without cracking. Additionally, the coatings thermal treatment at $1050^{\circ} \mathrm{C}$ allowed their partial sintering.

Keywords: fly ash, coatings, suspensions, zeta potential.

\section{INTRODUCCIÓN}

La ceniza volante es un material de desecho generado durante la combustión del carbón en las plantas carboeléctricas (1). Los residuos generados en la producción de energía eléctrica tienen diferentes aplicaciones en la industria de la construcción (2) y nuevas aplicaciones como materiales vitrocerámicos (3). Cerca de 500 millones de toneladas de ceniza volante son producidas anualmente en todo el mundo (4). En México, dos plantas de generación de energía localizadas en el norte de Coahuila, generan cerca de 1.62 millones de toneladas anualmente (5). La ceniza volante consiste principalmente de partículas amorfas con pequeñas cantidades de material cristalino y carbón sin quemar. En la fase cristalina de la ceniza volante se ha identificado mullita, cuarzo y hematita $(6,7,8)$.

La ceniza volante contiene esferas amorfas llamadas cenoesferas (9). Su bajo costo, su peso ligero y su baja conductividad térmica, las hacen apropiadas para aplicaciones como bloques aislantes y como recubrimientos para protección térmica de substratos (6). Existen diferentes procesos para obtener recubrimientos sobre substratos metálicos $(10,11)$. La deposición electroforética (EPD) es una buena opción debido a su bajo costo de inversión, a su velocidad de deposición rápida, a la uniformidad de los recubrimientos y a la facilidad de obtención de recubrimientos de sustratos de forma compleja (12). En este proceso, las partículas suspendidas en un líquido se mueven y depositan en un electrodo de trabajo al aplicarles un campo eléctrico (13).

El potencial zeta es un parámetro que permite evaluar, a través de la movilidad de las partículas en una suspensión, la estabilidad de una suspensión coloidal. Esta propiedad es importante para determinar las condiciones más adecuadas para obtener los recubrimientos electroforéticos. Todas las partículas en suspensión exhiben un potencial zeta o carga superficial. La solución que rodea a las partículas contiene iones de carga opuesta para mantener la neutralidad del sistema. Cuando las partículas tienen un valor grande de potencial zeta, positivo o negativo, tenderán a repelerse y 
la suspensión será estable. Si el valor de potencial zeta es pequeño, la tendencia a la floculación se incrementa. Si la carga neta en las partículas es cero (punto isoeléctrico), la suspensión es altamente inestable dificultándose su deposición electroforética (14).

Hasta hace algunos años, las mediciones de potencial zeta eran realizadas por medio de suspensiones de baja concentración de sólidos utilizando métodos ópticos. Con el desarrollo de la técnica electroacústica es ahora posible realizar mediciones en suspensiones con altas concentraciones de sólidos. En esta técnica, se calcula la movilidad a partir de ondas de sonido generadas por un campo eléctrico. El movimiento oscilatorio de las partículas resulta de la transferencia de momentum al líquido por ondas acústicas. Este efecto ha sido llamado Amplitud de Sonido Electrocinético (ESA), lo cual es exactamente análogo a la movilidad electroforética. La movilidad obtenida por el efecto electroacústico es la movilidad dinámica o de corriente alterna (AC) de la partícula (15). Su magnitud es la velocidad de la partícula por unidad de resistencia al campo. Esta técnica permite efectuar titulaciones automáticas y medir directamente el potencial zeta en soluciones con alta concentración. Por consecuencia, las mediciones pueden realizarse rápidamente en suspensiones sin necesidad de obtener muestras diluidas (15).

Esta investigación se enfocó en la caracterización de la ceniza volante y en su preparación para estudiar su estabilidad en términos de potencial zeta en diferentes medios polares y a diferentes $\mathrm{pH}$ utilizando la técnica electroacústica. El conocimiento del valor de la carga eléctrica superficial, de la movilidad y del potencial zeta de las partículas fue utilizado para determinar las mejores condiciones para la deposición electroforética de las partículas de ceniza volante sobre substratos de acero inoxidable 304.

\section{PARTE EXPERIMENTAL}

Las muestras de ceniza volante fueron obtenidas de una planta carboeléctrica localizada en Coahuila, México. La composición química de estas muestras fue determinada por medio de un equipo de absorción atómica de la marca Termo Elemental, Modelo Solar S4 y un equipo de espectroscopía por plasma de la marca Termo Elemental modelo Iris Intrepid II XSP. La preparación de las muestras se realizó con metaborato de litio a $1000^{\circ} \mathrm{C}$ y posteriormente se realizó la digestión ácida de la muestra. El contenido de carbón fue obtenido por medio de la técnica de combustión en un equipo de la marca LECO modelo CS-244 utilizando $1 \mathrm{~g}$ de muestra en polvo molida a malla \#100. Estos equipos de análisis están calibrados con soluciones estándar trazables a NIST. Las fases presentes en la ceniza volante fueron identificadas en un difractómetro de rayos X de la marca Phillips modelo X'Pert PW 3040 con goniómetro vertical. Los registros se efectuaron de 10 a $80^{\circ}(2 \theta)$ con un paso de $0.05^{\circ}(2 \theta)$, tiempo de conteo de $5 \mathrm{~s}$ y utilizando radiación de $\mathrm{Cu}$ y monocromador secundario (filtro de $\mathrm{Ni}$ ). Se realizaron estudios de análisis térmico diferencial (ATD) y de análisis termogravimétrico (ATG) en un equipo Perkin Elmer modelo Pyris Diamond con una velocidad de calentamiento de $10^{\circ} \mathrm{C} / \mathrm{min}$ en una atmósfera de aire, colocando la muestra en un crisol de platino y utilizando $\mathrm{Al}_{2} \mathrm{O}_{3}$ como material de referencia. Se utilizó una cantidad de $15 \mathrm{mg}$ de ceniza volante y el estudio se realizó desde temperatura ambiente hasta $1350^{\circ} \mathrm{C}$. La morfología de las partículas de ceniza fue analizada en un microscopio electrónica de barrido (MEB) de la marca Phillips modelo XL30 y ESEM equipado con sistema de análisis de espectrometría de energías dispersivas por EDXS, el cual fue operado a $20 \mathrm{kV}$ utilizando una distancia de trabajo de 9.8 y los polvos fueron depositados directamente en el portamuestras para su análisis. La eliminación del Fe fue realizada por medio de separación magnética en seco y en húmedo con imanes de neodimio y el $\mathrm{C}$ fue eliminado por medio de calcinación a $680^{\circ} \mathrm{C}$ durante 2 horas. Después de la eliminación de hierro y carbón se determinó la composición química de la ceniza y su distribución del tamaño de partícula. La ceniza volante fue preparada por molienda en un molino planetario de la marca Retsch modelo PM 200 adicionando gotas de ácido acético y utilizando tiempos de molienda de 1 a 15 horas y una velocidad de $400 \mathrm{rpm}$. La distribución del tamaño de partícula fue determinada por difracción láser en un equipo Coulter LS100Q equipado con módulo fluido de velocidad variable.

Las mediciones de potencial zeta fueron realizadas, por medio de un equipo Zetaprobe (Colloidal Dynamics), a suspensiones de $1 \%$ de ceniza volante en $300 \mathrm{ml}$ de agua, etanol, acetona e isopropanol. El ajuste del $\mathrm{pH}$ se realizó con adiciones de soluciones acuosas ( $1 \mathrm{M} \mathrm{HCl}$ y $1 \mathrm{M} \mathrm{NaOH})$. La suspensión fue mantenida en agitación constante durante los experimentos. Las mediciones de potencial zeta fueron graficadas en función del $\mathrm{pH}$. Todos los experimentos fueron realizados a temperaturas de $27 \pm 2^{\circ} \mathrm{C}$. Los ensayos de deposición electroforética fueron realizados usando acetona como medio de dispersión; voltajes de 100, 200 y 300 V y tiempos de deposición de 1 a 5 minutos. Adicionalmente se realizaron experimentos preliminares de sinterización a $1050^{\circ} \mathrm{C}$ durante 15 minutos en un horno de microondas de la marca Thermwave utilizando una velocidad de calentamiento de $30^{\circ} \mathrm{C} / \mathrm{min}$. Los recubrimientos obtenidos fueron analizados por MEB/EDXS en su superficie.

\section{RESULTADOS Y DISCUSIÓN}

El análisis químico de la ceniza volante es mostrado en la Tabla I. Sus componentes principales son el $\mathrm{SiO}_{2}$ y la $\mathrm{Al}_{2} \mathrm{O}_{3}$. Los contenidos de $\mathrm{Fe}_{2} \mathrm{O}_{3}$ y $\mathrm{C}$ son muy elevados para su aplicación como recubrimientos cerámicos por lo que deben ser reducidos a un mínimo. En la Figura 1 se muestra el difractograma de la ceniza volante. El espectro de difracción de rayos $X$ muestra una parte de naturaleza amorfa y una parte cristalina constituida principalmente por cuarzo y mullita. La Figura 2 muestra la fotomicrografía obtenida en MEB de la ceniza volante. Se observan partículas irregulares y esféricas con tamaños de 1 a $100 \mu \mathrm{m}$ con un tamaño promedio de partícula de $72.8 \mu \mathrm{m}$.

TABLA I. COMPOSICIÓN QUÍMICA DE LA CENIZA VOLANTE.

\begin{tabular}{|c|c|c|c|c|c|c|c|c|c|c|c|c|}
\hline \multicolumn{9}{|c|}{ Composición química (\% en peso) } & & Humedad & $\begin{array}{l}\text { Pérdida por } \\
\text { calcinación }\end{array}$ & Otros \\
\hline $\mathrm{SiO}_{2}$ & $\mathrm{Al}_{2} \mathrm{O}_{3}$ & $\mathrm{~K}_{2} \mathrm{O}$ & $\mathrm{MgO}$ & $\mathrm{TiO}_{2}$ & $\mathrm{CaO}$ & $\mathrm{Fe}_{2} \mathrm{O}_{3}$ & $\mathrm{Na}_{2} \mathrm{O}$ & C & S & \multirow{2}{*}{0.25} & \multirow{2}{*}{1.09} & \multirow{2}{*}{0.132} \\
\hline 60.11 & 23.52 & 0.168 & 0.95 & 0.63 & 3.29 & 6.35 & 0.16 & 3.35 & 0.216 & & & \\
\hline
\end{tabular}




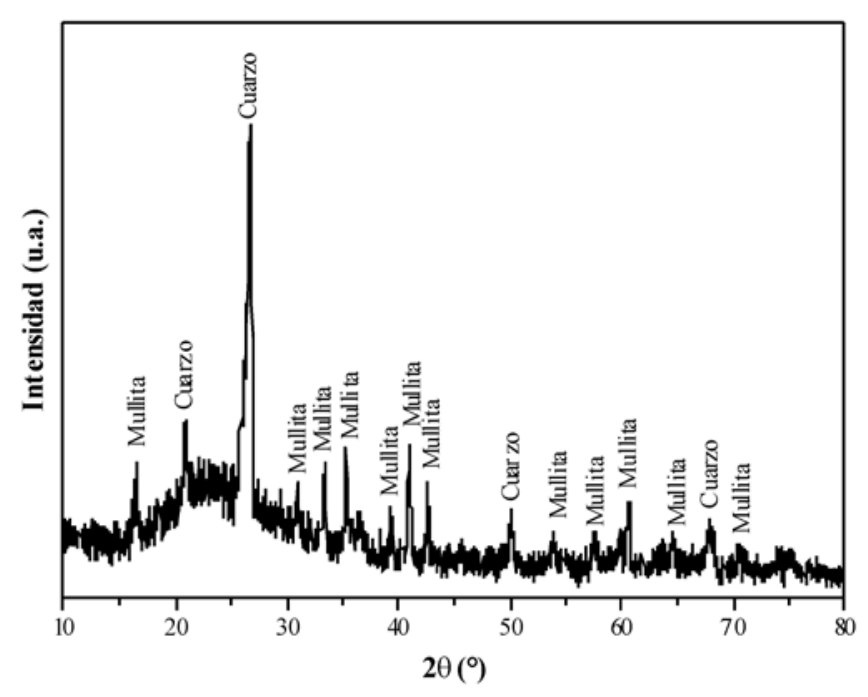

Fig. 1- Difractograma de la ceniza volante.

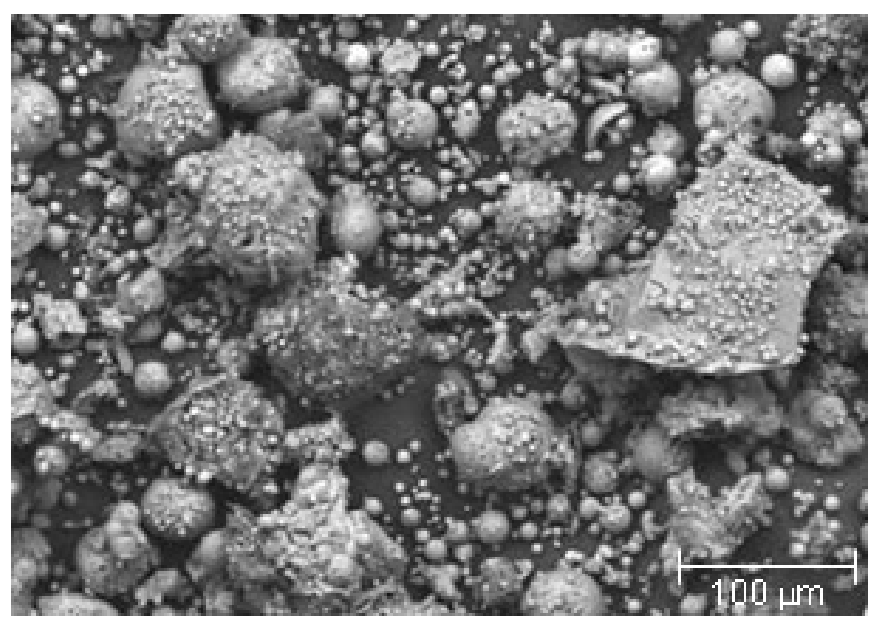

Fig. 2- Fotomicrografía obtenida por MEB de la ceniza volante.

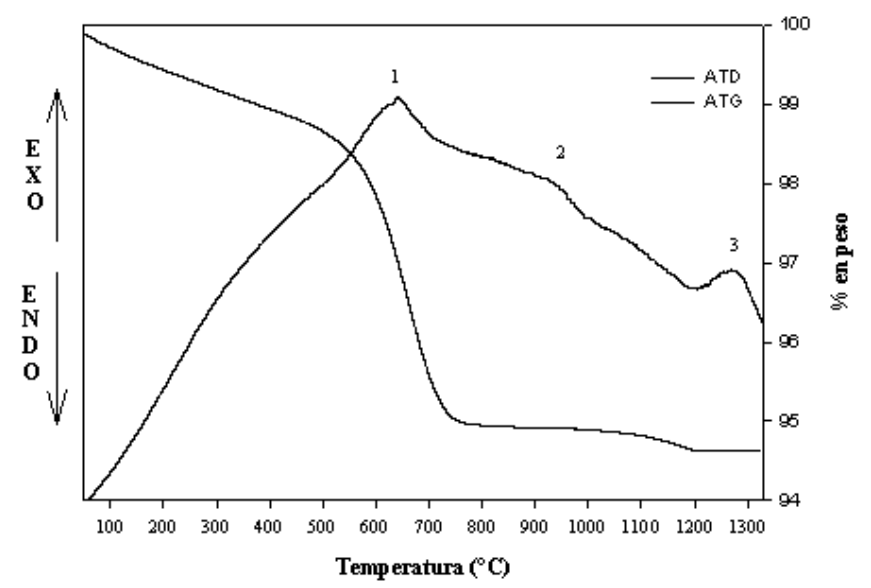

Fig. 3- Curva de ATD y ATG de la ceniza en un rango de temperatura de $100-1350^{\circ} \mathrm{C}$.
En la Figura 3 se muestra la curva de ATD / ATG obtenida de la muestra de ceniza volante. Los principales cambios que ocurren en la ceniza volante con el incremento de temperatura son la pérdida de carbón y algunas transformaciones de fase. El primer pico exotérmico presente en la curva de ATD a aproximadamente $600^{\circ} \mathrm{C}$ (No. 1) corresponde a la combustión del carbón. El segmento mostrado en la curva ATD con el No. 2 corresponde a la transformación del cuarzo a tridimita la cual inicia a una temperatura aproximada de $860^{\circ} \mathrm{C}$. Posteriormente el pico mostrado a aproximadamente $1200^{\circ} \mathrm{C}$ (No. 3) representa la cristalización de la mullita (16). Por medio de la curva de ATG se puede observar que la muestra de ceniza volante es estable a temperaturas mayores a $700^{\circ} \mathrm{C}$. Durante el calentamiento se observa la eliminación del carbón residual en el intervalo de temperatura de 450 a $700^{\circ} \mathrm{C}$. A temperaturas inferiores a $400^{\circ} \mathrm{C}$, se presenta una pérdida en peso atribuida a la eliminación de humedad.

El análisis térmico permitió determinar las condiciones óptimas para eliminar el carbón y las temperaturas a las que ocurren cambios de fase de los compuestos presentes en la ceniza. En la Tabla II se muestra la composición química de la ceniza después de un tratamiento térmico a $680^{\circ} \mathrm{C}$ y de las separaciones magnéticas en seco y en húmedo. El contenido de Fe en la ceniza fue reducido a $0.30 \%$ en peso y el contenido de C disminuyó a $0.068 \%$ en peso. También se puede observar que el contenido de $\mathrm{S}$ disminuyó considerablemente. Después de estos tratamientos de limpieza, el material fue molido durante 15 horas hasta obtener un tamaño promedio de partícula de $1.5 \mu \mathrm{m}$ para poder realizar los ensayos de deposición electroforética. La Figura 4 muestra la distribución de tamaño de partícula de la ceniza volante después de los tratamientos realizados en función del tiempo de molienda.

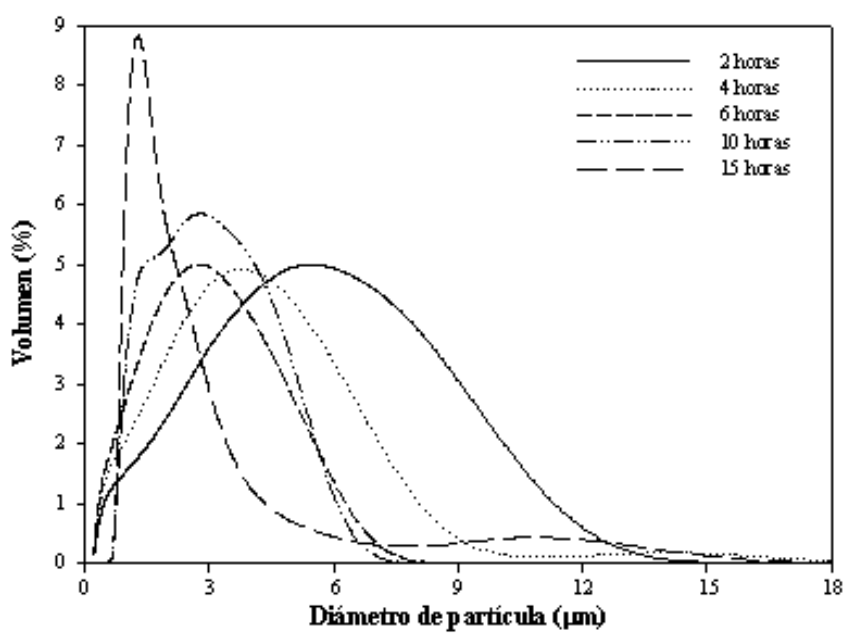

Fig. 4- Distribución de tamaño de partícula de la ceniza volante a diferentes tiempos de molienda después de la eliminación del Fe y C.

TABLA I. COMPOSICIÓN QUÍMICA DE LA CENIZA DESPUÉS DE LOS TRATAMIENTOS DE LIMPIEZA.

\begin{tabular}{|c|c|c|c|c|c|c|c|c|c|c|c|}
\hline \multicolumn{9}{|c|}{ Composición química (\% en peso) } & \multicolumn{2}{c|}{ Humedad } & \multicolumn{2}{c|}{ Pérdida por calcinación } \\
\hline $\mathrm{SiO}_{2}$ & $\mathrm{Al}_{2} \mathrm{O}_{3}$ & $\mathrm{~K}_{2} \mathrm{O}$ & $\mathrm{MgO}$ & $\mathrm{TiO}_{2}$ & $\mathrm{CaO}$ & $\mathrm{Fe}_{2} \mathrm{O}_{3}$ & $\mathrm{Na}_{2} \mathrm{O}$ & $\mathrm{C}$ & $\mathrm{S}$ & \\
\hline 6 & 28.05 & 0.72 & 0.86 & 0.87 & 2.24 & 0.30 & 0.64 & 0.068 & 0.027 & $<0.01$ \\
\end{tabular}


En la Figura 5 se muestra la curva de movilidad dinámica en función del $\mathrm{pH}$ para suspensiones de ceniza volante en diferentes medios. La mayor movilidad ocurrió en agua (-3.8 $\mathrm{m}^{2} / \mathrm{Vs}$ a un $\mathrm{pH}$ de 10.5). Sin embargo, el uso del agua como medio de dispersión está limitado a bajos voltajes debido a la electrólisis que se presenta en los electrodos al aplicar una diferencia de potencial a la suspensión. Después del agua, la suspensión de ceniza volante en acetona-solución acuosa de $\mathrm{NaOH}$ presentó las mejores condiciones para la deposición electroforética. La Figura 6 muestra la curva de potencial zeta en función del $\mathrm{pH}$ de esta suspensión. A un $\mathrm{pH}$ de aproximadamente 9 se tiene el valor más alto de potencial zeta $(-50 \mathrm{mV})$ y por consecuencia las mayores fuerzas repulsivas entre partículas y la mayor estabilidad de la suspensión. A mayores adiciones de solución acuosa de $\mathrm{NaOH}$, se observó una disminución en el valor del potencial zeta. Este efecto podría ser explicado considerando una disminución de las fuerzas repulsivas entre las partículas debido a la compresión de la doble capa eléctrica formada cerca de las partículas (17). El punto isoeléctrico de la suspensión ocurrió a un $\mathrm{pH}$ de 6.5, el cual es similar al punto isoeléctrico de la mullita (18).

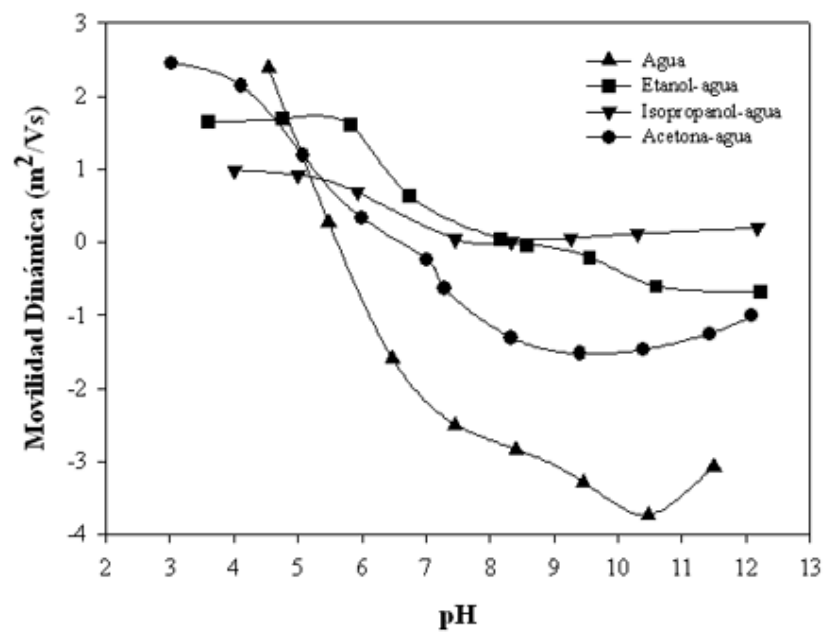

Fig. 5- Movilidad dinámica Vs. pH para suspensiones de ceniza volante en diferentes medios.

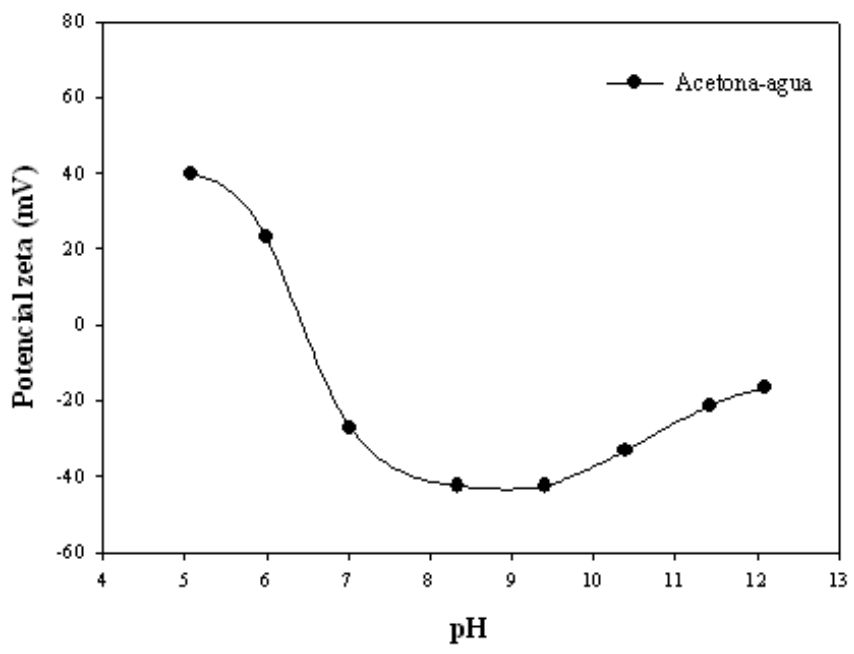

Fig. 6- Potencial zeta Vs. pH para suspensiones de ceniza volante en acetona con adición de soluciones acuosas de $\mathrm{HCl}$ y $\mathrm{NaOH}$.
La adición de soluciones acuosas de $\mathrm{NaOH}$ y $\mathrm{HCl}$ a la suspensión de ceniza volante en acetona permitió la modificación de la carga superficial de la ceniza volante por medio de una adsorción preferencial de iones. Para este sistema, los principales componentes que constituyen la ceniza volante y que determinan la carga superficial son el $\mathrm{SiO}_{2}$ y la $\mathrm{Al}_{2} \mathrm{O}_{3}$. La adsorción de iones $\mathrm{H}^{+}$produce una superficie cargada positivamente a bajos $\mathrm{pH}$ mientras que la adsorción de iones $\mathrm{OH}^{-}$lleva a la formación de una superficie cargada negativamente a alto $\mathrm{pH}$ como se muestra en las Figuras 5 y 6 . La formación de cargas positivas o negativas en la superficie de las partículas puede ser esquematizada por las siguientes reacciones (18):
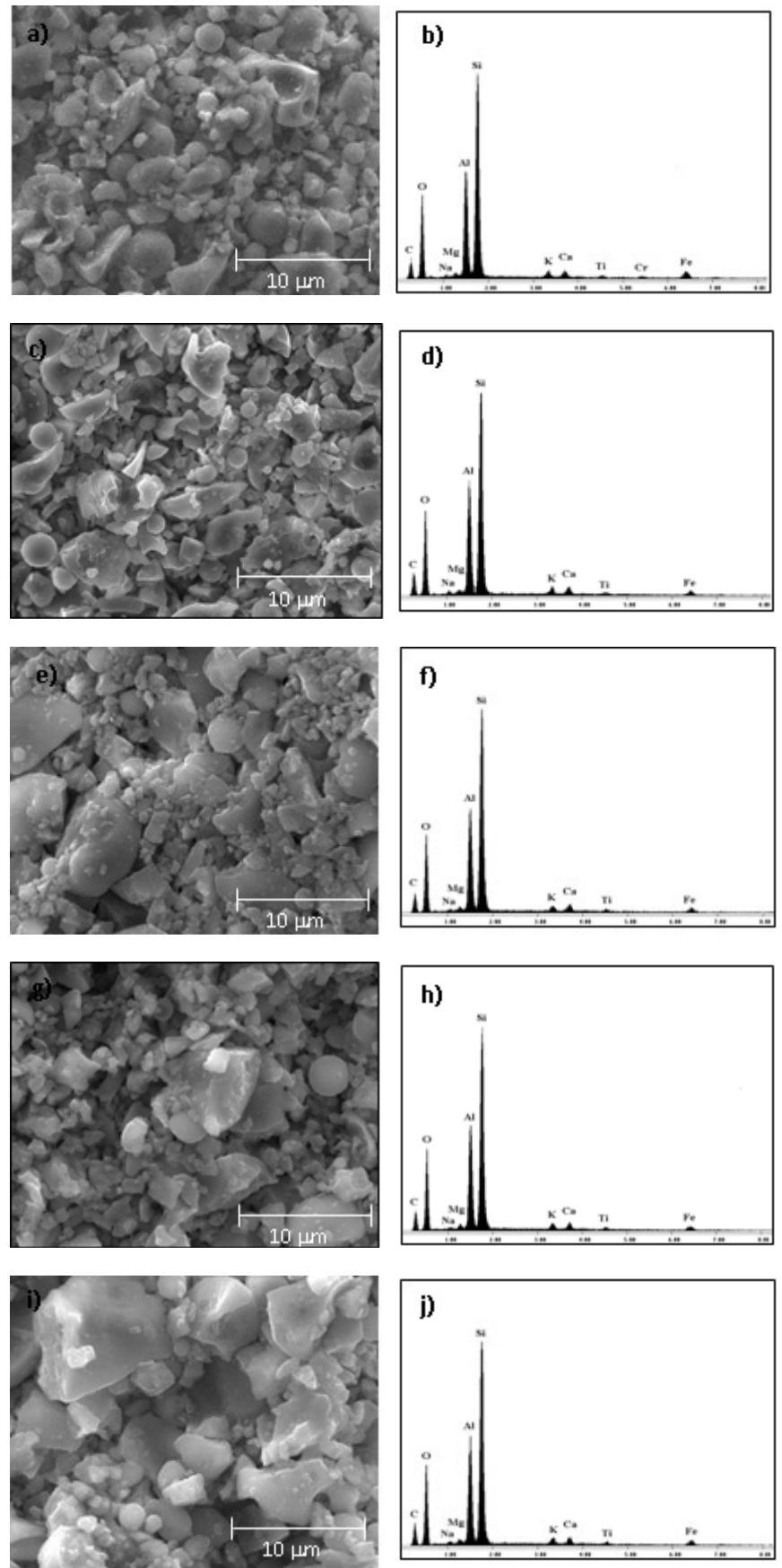

Fig. 7- Recubrimientos de ceniza volante obtenidos a $100 \mathrm{~V}$ y 1(a), 2(c), $3(\mathrm{e}), 4(\mathrm{~g})$ y $5(\mathrm{i})$ minutos de deposición con su respectivo espectro de composición química $(b, d, f, h, j)$. 
A bajo $\mathrm{pH}$

$\mathrm{SiOH}+\mathrm{H}^{+} \rightarrow \mathrm{SiOH}_{2}^{+}$

$\mathrm{AlOH}+\mathrm{H}^{+} \rightarrow \mathrm{AlOH}_{2}^{+}$

A pH elevado

$\mathrm{SiOH}+\mathrm{OH}^{-} \rightarrow \mathrm{SiO}^{-}+\mathrm{H}_{2} \mathrm{O}$

$\mathrm{AlOH}+\mathrm{OH}^{-} \rightarrow \mathrm{AlO}^{-}+\mathrm{H}_{2} \mathrm{O}$

Por lo tanto, para los experimentos de deposición electroforética se prepararon las suspensiones de ceniza en acetona con adición de solución acuosa de $\mathrm{NaOH}$ a un $\mathrm{pH}$ de 9 (valor de potencial zeta de $-50 \mathrm{mV}$ ) y el material fue depositado en el ánodo. La Figura 7 muestra las fotomicrografías de los recubrimientos obtenidos por la técnica de deposición electroforética a $100 \mathrm{~V}$ con 1(a), 2(c), 3(e), 4(g) y 5(i) minutos de deposición y su respectivo espectro de composición química obtenido por EDX. En esta Figura es posible observar recubrimientos uniformes con buen empaquetamiento y un aumento en la cantidad y tamaño de los aglomerados al incrementar el tiempo de deposición. La composición química de los recubrimientos contiene principalmente Si y $\mathrm{Al}$ con algunas cantidades pequeñas de $\mathrm{Ca}, \mathrm{K}, \mathrm{Na}$, $\mathrm{Ti}$, etc. Solamente en el espectro de $100 \mathrm{~V}$ y 1 minuto de deposición se muestran picos correspondientes al $\mathrm{Cr}$ y al Fe ligeramente más alto que los recubrimientos obtenidos a tiempos de deposición mayores, los cuales están relacionados con el espesor más delgado del recubrimiento. La Figura 8 muestra que la cantidad de material depositado en los substratos de acero inoxidable varió de $20 \mathrm{mg} / \mathrm{cm}^{2}$ (100 V y 1 minuto) a $80 \mathrm{mg} /$ $\mathrm{cm}^{2}$ (300 V y 5 minutos).

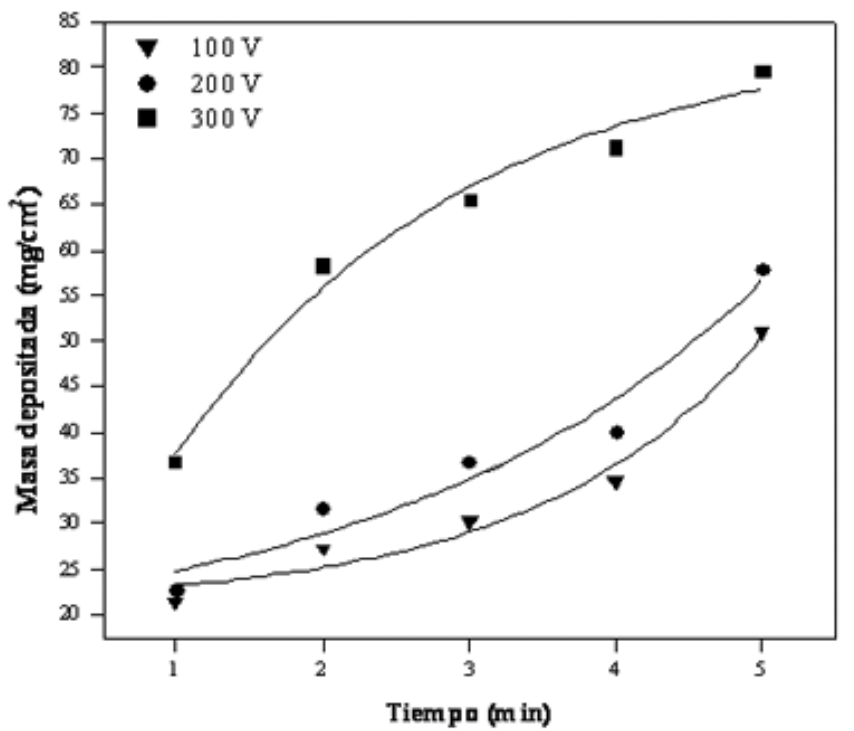

Fig. 8- Cantidad de ceniza volante depositada en función del tiempo y del voltaje aplicado.

La Figura 9 muestra fotomicrografías a dos magnificaciones de la morfología de un recubrimiento de ceniza con bajo contenido de Fe y C obtenido por deposición electroforética a $200 \mathrm{~V}$ y 1 minuto y sinterizado por microondas a $1050^{\circ} \mathrm{C}$ durante 15 minutos. Como se puede observar en la Fig. 9(a) se muestra el recubrimiento con algunas partículas con morfología de fibras en su superficie las cuales fueron identificadas como mullita de acuerdo al espectro de composición química determinado por EDXS (Figura 9(b)). En la fotomicrografía de la Figura 9(c) se observa el mismo recubrimiento a una mayor magnificación donde se puede distinguir el inicio de la sinterización ya que se tiene la formación de cuellos entre algunas partículas. Finalmente la Figura 9(d) muestra el espectro EDXS de este recubrimiento.
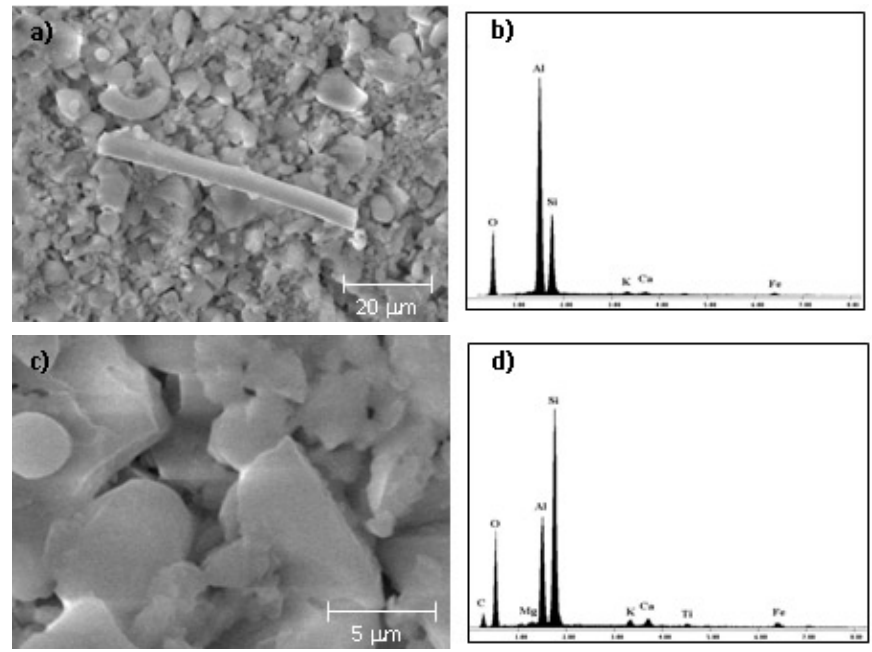

Fig. 9- Fotomicrografías del recubrimiento sinterizado a $1050^{\circ} \mathrm{C}$ (a) y (c) con su espectro de composición química (b y d).

\section{CONCLUSIONES}

Mediante una separación magnética y un tratamiento térmico a $680{ }^{\circ} \mathrm{C}$ a la ceniza volante fue posible reducir los contenidos de Fe y C a 0.30 y $0.068 \%$ en peso, respectivamente.

Las mediciones de movilidad electroforética en agua, etanol, isopropanol y acetona mostraron que el medio más adecuado para obtener suspensiones estables de ceniza fue una solución de acetona-solución acuosa (el valor más elevado de potencial zeta fue de $-50 \mathrm{mV}$ a un $\mathrm{pH}$ de 9 ajustado mediante una adición de $\mathrm{NaOH}$ ).

Los ensayos de deposición electroforética mostraron que en la medida en que se incremente el voltaje y el tiempo de deposición se logran mayores depósitos $\left(20 \mathrm{mg} / \mathrm{cm}^{2}\right.$ aplicando $100 \mathrm{~V}$ durante 1 minuto hasta $80 \mathrm{mg} / \mathrm{cm}^{2}$ al aplicar $300 \mathrm{~V}$ durante 5 minutos). Sin embargo, al incrementarse estos dos parámetros se incrementa el tamaño de los aglomerados lo cual puede afectar la temperatura de sinterización. En este trabajo, el tratamiento térmico preliminar a $1050^{\circ} \mathrm{C}$ permitió obtener un sinterizado parcial de los recubrimientos, lo cual servirá de base para realizar la siguiente parte de esta investigación.

\section{AGRADECIMIENTOS}

Los autores del presente trabajo agradecen al Consejo Nacional de Ciencia y Tecnología (Proyecto Conacyt-DAIC Clave 44902) por el apoyo económico brindado.

\section{BIBLIOGRAFÍA}

1. A. Baba, A. Kaya, Leaching characteristics of fly ash from thermal power plants of Soma and Tuncbilek, Turkey, Environ. Monit. Assess. 91:171-181 (2004).

2. A. Acosta, I. Iglesias, M. Aineto, M. Romero, J. Ma. Rincón, Utilisation of IGCC slag and clay steriles in soft mud bricks for use in building bricks manufacturing, Waste Manag. 22(8) 887-891 (2002). 
3. M. Aineto, A. Acosta, Las escorias de la central térmica GICC ELCOGAS como materia prima para la síntesis de materiales vitrocerámicos Parte 1: Comportamiento en fusión de las escorias GICC y obtención del vidrio original, Bol. Soc. Esp. Ceram. V. 44 [6] 399-404 (2005).

4. P.K. Mehta, Concrete Technology for Sustainable Development, Concr. Int. 21[11] 47-53 (1999).

5. B. Treviño, I. Gomez, Obtención de fases del cemento utilizando desechos agrícolas e industriales, Ciencia UANL, V. 5[002] 190-196 (2002).

6. L. K. A. Sear, The properties and use of coal fly ash, Chapter 1, The production and properties of fly ash, Thomas Telford Publishing, UK, 2001.

7. S. Khanra, D. Mallick, S. N. Dutta, S. K. Chaudhuri, Studies on the phase mineralogy and leaching characteristics of coal fly ash, Water Air Soil Pollut. 107:251-275 (1998).

8. S. Gomes, M. François, Characterization of mullite in silicoaluminous fly ash by XRD, TEM and ${ }^{29}$ Si MAS NMR, Cement Concr. Res. 30, 175-181 (2000).

9. D. C. Adriano, A.L. Page, A.A. Elseewi, A.C. Chang, I. Straughan, Utilization and disposal of fly ash and other coal residues in terrestrial ecosystems: A review, J. Environ. Qual., 9:333-344 (1980).

10. A. Salazar, J. Gómez-García, P. Poza, V. Utrila, Degradación de recubrimientos $\mathrm{ZrO} 2-\mathrm{CaO} / \mathrm{NiAlMo}$ por oxidación isoterma, Bol. Soc. Esp. Ceram. V. 45[6] 389-395 (2006)
11. J. Gómez-García, P. Poza, V. Utrilla, Crecimiento y caracterización de recubrimientos cerámicos con aplicaciones como barreras térmicas, Bol. Soc Esp. Ceram. V. 45[2] 70-74 (2006).

12. S. Put, J. Vleugels, O. Van der Biest, Functionally graded WC-Co materials produced by electrophoretic deposition Scripta Mater. 45(10):1139-45 (2001)

13. O. O. Van der Biest, L. J. Vandeperre, Electrophoretic deposition of materials, Annu. Rev. Mater. Sci., 29:327-52 (1999)

14. S. Usui, DLVO Theory of Colloid Stability, In: Ohshima H., Furusawa K. (Eds.), Surfactant science series, V. 76: Electrical Phenomenon at Interfaces. Fundamentals, Measurements and Applications, $2^{\text {nd }}$ Edition, New York, Basel (1980).

15. R. W. O'Brien, D. V. Cannon, W. N. Rowlands, “Electroacoustic determination of particle size and zeta potential", J. Colloid Interface Sci. 173:406-418 (1995).

16. M. Aineto, A. Acosta, J. M. Rincón, M. Romero, Thermal expansion of slag and fly ash from coal gasification in IGCC power plant, Fuel, 85(16):23522358 (2006).

17. M. Elimelech, C.R. O'Melia, Effect of Electrolyte Type on the Electrophoretic Mobility of Polystyrene Latex Colloids, Colloid Surface, 44:165-178 (1990).

18. M.N. Rahaman, Science of colloidal processing, pp. 146-200, Ceramic processing and sintering, Ed. Marcel Dekker, Inc., (USA) 1998. 